\section{Anomalias congênitas na perspectiva dos determinantes sociais da saúde}

\section{Congenital anomalies from the perspective of social determinants of health}

\section{Anomalías congénitas desde la perspectiva de los determinantes sociales en salud}

\section{Resumo}

O objetivo deste estudo foi analisar os fatores associados aos casos de anomalias congênitas na perspectiva dos determinantes sociais da saúde no Estado do Rio Grande do Sul, Brasil. Trata-se de um estudo do tipo caso-controle, com todas as duplas de mães e nascidos vivos no período de 2012 a 2015 no estado, sendo considerado o total de nascidos vivos com anomalia congênita (5.250), e realizada amostragem aleatória de 21 mil sem anomalia congênita, conforme as informações das Declarações de Nascidos Vivos. Para análise estatística, foram realizados testes qui-quadrado e modelos de regressão logística com o SPSS. O modelo de Dahlgren es Whitehead foi utilizado como base para agrupamento e discussão das variáveis. No modelo multivariado, todas variáveis que se mostraram associadas significativamente com o desfecho foram no sentido de aumentar a chance de nascimentos com anomalia congênita: as mulheres pretas tiveram 20\% mais chance, comparadas às brancas $(O R=1,20$; valor de $p=0,013)$; ter mais de 40 anos aumentou em 97\% a chance, quando comparadas às de 18 a 29 anos; as mulheres com menos de quatro anos de estudo apresentaram 50\% mais chance, comparadas às mulheres com 12 anos ou mais de estudo $(O R=1,50$; valor de $p=0,001)$; as mulheres que não realizaram nenhuma consulta de pré-natal tiveram 97\% mais chance, comparadas às mulheres que realizaram sete ou mais consultas $(O R=1,97$; valor de $p=0,001) ;$ e a ocorrência de abortos/perdas fetais aumentou em 17\% a chance, em relação a nunca ter tido abortos/perdas fetais prévios $(O R=1,17$; valor de $p=0,001)$. Os resultados trazem à discussão as desigualdades raciais e sociais, relacionando-as às iniquidades em saúde.

Anormalidades Congênitas; Determinantes Sociais; Fatores Raciais; Racismo
Graziella Chaves Trevilato 1

Deise Lisboa Riquinho 1

Marilise Oliveira Mesquita 1

Idiane Rosset 1

Lia Giraldo da Silva Augusto 2

Luciana Neves Nunes 2

doi: 10.1590/0102-311X00037021

\section{Correspondência}

G. C. Trevilato

Universidade Federal do Rio Grande do Sul.

Rua São Manoel 963, Porto Alegre, RS 90620-110, Brasil.

grazitrevilato.enfermagem@gmail.com

1 Universidade Federal do Rio Grande do Sul, Porto Alegre,

Brasil.

2 Instituto Aggeu Magalhães, Fundação Oswaldo Cruz, Recife, Brasil. 


\section{Introdução}

As anomalias congênitas podem ser definidas como alterações estruturais ou funcionais que ocorrem durante a vida intrauterina. Tais ocorrências se configuram em importantes agravos geradores de morte na infância, doenças crônicas e deficiências que podem ser identificadas no pré-natal, no parto ou ao longo da vida ${ }^{1}$. Aproximadamente uma em cada cinco mortes nos primeiros 28 dias de vida são decorrentes de defeitos congênitos na América Latina e no Caribe 2. Cerca de 50\% das anomalias congênitas não estão ligadas a um condicionante específico, entretanto existem algumas causas conhecidas que podem estar relacionadas, como infecções, estado nutricional materno e fatores ambientais, genéticos, demográficos e socioeconômicos 1 .

A baixa renda pode ser um determinante indireto de anomalias congênitas, ocorrendo com maior frequência entre famílias e países com recursos econômicos limitados. Aproximadamente $94 \%$ das anomalias congênitas graves ocorrem em países de baixa a média renda, o que pode estar relacionado à dieta inapropriada das gestantes, a aumento da exposição a infecções e/ou álcool, ou acesso precário a cuidados de saúde, inclusive falta de consultas de pré-natal ou a realização do pré-natal de forma inadequada 1.

Nesse contexto ampliado, o modelo de Dahlgren \& Whitehead 3 aborda os determinantes sociais da saúde (DSS) como fatores sociais, econômicos, culturais, étnicos/raciais, psicológicos e comportamentais que podem influenciar na ocorrência de problemas de saúde e seus fatores de risco na população. Os DSS são dispostos em cinco camadas dentro do modelo, sendo a primeira relacionada às características biológicas individuais, a segunda referente aos fatores sobre estilo de vida e comportamentos individuais, a terceira relacionada às redes comunitárias e de apoio social, a quarta referente às condições de vida e trabalho, seguida da quinta e última camada, que abrange os fatores relacionados às condições sociais, econômicas, culturais e ambientais da sociedade.

Maeyama et al. 4 abordam os DSS, particularmente a partir do modelo de Dahlgren \& Whitehead 3, numa dimensão macro e microssocial. Na dimensão macrossocial, o modelo demonstra a relação existente entre as desigualdades em saúde geradas pelas iniquidades sociais e como a análise destas variáveis pode subsidiar a construção de políticas públicas que possibilitem as mesmas oportunidades a todas as pessoas. No nível microssocial, os autores sugerem a intervenção dos profissionais da saúde mediante articulações interinstitucionais e intersetoriais, com a finalidade de facilitar o acesso aos serviços 4 . O tema se amplia para a determinação social como produto e produtora de agravos à saúde a partir das condições de vida e trabalho, ressaltando que a saúde vai além de um fato biológico-natural, sendo influenciada positiva ou negativamente pelo contexto social e a história de vida individual e da coletividade 5 .

Ao considerar como injustas as causas sociais que levam ao adoecimento e à morte, é evidenciada a necessidade urgente de transformação e compreensão das iniquidades em saúde para estabelecer o cuidado adequado e a dimensão coletiva de tais agravos, especialmente pelo desafio de promover saúde em um país tão desigual como o Brasil 6. Nesse sentido, o Relatório Anual das Desigualdades Raciais no Brasil: 2009-20107 mostrou maiores chances de morte materna e infantil e menor acesso aos serviços de saúde pela população negra, além de diminuição da qualidade e, também, da expectativa de vida desse grupo racial. Essa é uma temática de extrema relevância para a saúde pública e ainda negligenciada no Brasil. Assim, o objetivo deste estudo foi analisar os fatores associados aos casos de anomalias congênitas na perspectiva dos DSS no Rio Grande do Sul, Brasil.

\section{Método}

Realizou-se um estudo do tipo caso-controle 8. A população do estudo compreendeu todas as duplas de mães e nascidos vivos no período de 2012 a 2015 no Rio Grande do Sul. Optou-se por iniciar a coleta de dados a partir do ano de 2012, pois, em 2011, ocorreram modificações no modelo de Declaração de Nascido Vivo (DNV), portanto, a partir de 2012, passou a ser utilizado o novo formulário 9. Foram incluídas no estudo apenas as DNV com município de residência materna no Rio Grande do Sul e, dentre estas, foram excluídas as DNV em que o campo referente à presença de anomalias congênitas no formulário não estava preenchido. 
O cálculo do tamanho da amostra foi realizado no programa WinPEPI, versão 11.43 (http://www. brixtonhealth.com/pepi4windows.html), com com base nos dados do DATASUS (Departamento de Informática do SUS). Em relação à amostra, para um nível de 5\% de significância, poder de 80\%, uma incidência estimada de notificação de anomalias congênitas em 1\%, e um odds ratio (OR) estimado em 1,5 para as características sociodemográficas, com proporção de 1:4 casos e controles, respectivamente, obteve-se o total mínimo de 4.730 nascidos vivos com anomalias e 18.920 nascidos vivos sem anomalias congênitas. Entretanto, como o número da amostra com anomalia congênita ficou muito próximo ao número total de anomalias da população, optou-se por estudar todos os casos com anomalias congênitas do período estudado, totalizando 5.250 nascidos vivos com anomalia congênita, ou seja, a amostra de casos correspondeu ao total dessa população, e 21 mil sem anomalia congênita, respeitando a proporção de 1:4 casos e controles. A coleta dos dados sociodemográficos e de anomalias congênitas foi obtida pelo Sistema de Informações sobre Nascidos Vivos do Estado do Rio Grande do Sul (SINASC/RS), e as variáveis maternas analisadas foram: faixa etária, escolaridade, raça/cor, município de residência, número de consultas de pré-natal, número de filhos vivos e número de abortos/perdas fetais em gestações anteriores.

Os dados coletados das DNV foram agrupados e descritos com base na classificação dos DSS do modelo de Dahlgren \& Whitehead 3, e as variáveis foram categorizadas como determinantes individuais, proximais e distais. Para tanto, neste estudo, foram considerados como determinantes individuais a faixa etária e a raça/cor (características biológicas individuais - primeira camada do modelo); como determinantes proximais, a quantidade de filhos vivos, a quantidade de abortos ou perdas fetais e o número de gestações anteriores (estilo de vida e comportamentos individuais, e redes comunitárias e de apoio social - segunda e terceira camadas do modelo); e, como determinantes distais, a escolaridade e o número de consultas de pré-natal (condições de vida e condições sociais - quarta e quinta camadas do modelo), a fim de melhor distribuir os dados disponíveis nas DNV.

Utilizou-se o programa estatístico SPSS, versão 18.0 (https://www.ibm.com/), para a realização da análise estatística. Os modelos de regressão logística univariado e multivariado foram aplicados para avaliar os fatores de risco para anomalias congênitas. As inclusões das variáveis no modelo multivariado foram realizadas a partir dos resultados das análises univariadas com critério de nível de significância de $20 \%$, ou seja, quando a variável independente se mostrou associada significativamente com o desfecho ao nível de 0,20 de significância, esta foi incluída no modelo multivariado. Os fatores em estudo foram as características sociodemográficas maternas (variáveis independentes) e as anomalias congênitas (variável dependente) foram o desfecho. A medida de efeito utilizada foi o OR complementado pelo intervalo de 95\% de confiança (IC95\%) e o critério adotado para a decisão sobre a permanência no modelo multivariado foi de nível de 5\% de significância. Para verificar o efeito da variável cor/raça sobre as variáveis escolaridade e número de consultas pré-natais, utilizou-se o teste qui-quadrado e, neste caso, também adotou-se o critério de nível de 5\% de significância.

Este projeto foi aprovado pelos Comitês de Ética em Pesquisa da Universidade Federal do Rio Grande do Sul (CAEE 84275318.4.0000.5347) e da Escola de Saúde Pública do Rio Grande do Sul (CAEE 84275318.4.3001.5312).

\section{Resultados}

Os casos de anomalias congênitas representaram 0,93\% do total de nascimentos de 2012 a 2015 no Rio Grande do Sul e o tipo de anomalias congênitas mais prevalente foi as malformações e deformidades congênitas do sistema osteomuscular, representando 45,4\% do total de anomalias. De acordo com as informações das DNV analisadas, 80,5\% das mães de recém-nascidos com anomalia congênita se autodeclararam de cor branca, enquanto 9,9\% se declararam de cor parda, seguida por 9,1\% de cor preta, 0,2\% de cor amarela e 0,3\% indígena. Já no grupo-controle (mães de recém-nascidos sem anomalias congênitas), $82,7 \%$ se autodeclararam de cor branca, 9,4\% de cor parda, 7,3\% de cor preta, $0,1 \%$ de cor amarela e $0,5 \%$ indígena. A variável raça/cor foi associada à ocorrência de anomalias congênitas (valor de $\mathrm{p}<0,001$ ) (Tabela 1). 
Tabela 1

Características sociodemográficas e de saúde de mães de nascidos vivos, segundo os grupos de casos e controles. Porto Alegre, Rio Grande do Sul, Brasil, 2012-2015 ( $n=26.000)$.

\begin{tabular}{|c|c|c|c|c|c|c|}
\hline Variável & Casos & $\%$ & Controles & $\%$ & OR (IC95\%) & Valor de p \\
\hline Raça/Cor & & & & & & $<0,001$ \\
\hline Branca & 4.149 & 80,6 & 17.028 & 82,7 & 1,00 & \\
\hline Preta & 468 & 9,1 & 1.498 & 7,3 & $1,28(1,15-1,43)$ & \\
\hline Amarela & 8 & 0,2 & 20 & 0,1 & $1,64(0,72-3,72)$ & \\
\hline Parda & 509 & 9,9 & 1.938 & 9,4 & $1,08(0,97-1,19)$ & \\
\hline Indígena & 18 & 0,3 & 96 & 0,5 & $0,77(0,46-1,37)$ & \\
\hline Faixa etária (anos) & & & & & & $<0,001$ \\
\hline$<18$ & 406 & 7,7 & 1.520 & 7,2 & $1,14(1,01-1,28)$ & \\
\hline $18-29$ & 2.719 & 51,8 & 11.561 & 55,1 & 1,00 & \\
\hline $30-40$ & 1.931 & 36,8 & 7.512 & 35,8 & $1,09(1,02-1,17)$ & \\
\hline$>40$ & 193 & 3,7 & 406 & 1,9 & $2,02(1,69-2,41)$ & \\
\hline Anos de estudo & & & & & & $<0,001$ \\
\hline$<4$ & 158 & 3 & 512 & 2,4 & $1,52(1,25-1,84)$ & \\
\hline $4-7$ & 1.300 & 25 & 4.703 & 22,5 & $1,36(1,24-1,49)$ & \\
\hline 8-11 & 2.841 & 54,6 & 11.252 & 53,8 & $1,24(1,14-1,35)$ & \\
\hline 12 ou mais & 906 & 17,4 & 4.453 & 21,3 & 1,00 & \\
\hline Consultas de pré-natal & & & & & & $<0,001$ \\
\hline Nenhuma & 166 & 3,2 & 400 & 1,9 & $1,83(1,52-2,19)$ & \\
\hline $1-3$ & 408 & 7,8 & 1.090 & 5,2 & $1,65(1,46-1,86)$ & \\
\hline $4-6$ & 1.152 & 22 & 4.036 & 19,3 & $1,26(1,16-1,35)$ & \\
\hline 7 e mais & 3.502 & 67 & 15.399 & 73,6 & 1,00 & \\
\hline Abortos/Perdas fetais & & & & & & $<0,001$ \\
\hline Sim & 999 & 19,5 & 3.274 & 16,2 & $1,26(1,16-1,36)$ & \\
\hline Não & 4.128 & 80,5 & 16.994 & 83,8 & 1,00 & \\
\hline
\end{tabular}

IC95\%: intervalo de 95\% de confiança; OR: odds ratio.

A faixa etária predominante da mãe em ambos os grupos (casos e controles) foi de 18 a 29 anos, com $51,8 \%$ e 55,1\%, respectivamente, sendo que, no grupo de casos, a prevalência da faixa etária acima de 40 anos foi de 3,7\%, enquanto, no grupo controle, foi de 1,9\%, sendo essa variável associada à ocorrência de anomalias congênitas (valor de $\mathrm{p}<0,001$ ) (Tabela 1). Na análise preliminar, houve uma associação significativa da faixa etária < 18 anos com anomalias congênitas. Entretanto, na análise multivariada, essa associação não se mostrou significativa (IC95\%: 0,90; 1,18).

Quanto à escolaridade, a categoria de 8 a 11 anos de estudo foi predominante tanto no grupo de casos $(54,6 \%)$ quanto no grupo de controles $(53,8 \%)$, entretanto esta variável se mostrou associada significativamente com a ocorrência de anomalias congênitas (valor de $\mathrm{p}<0,001$ ). Todas as categorias de escolaridade mostraram chance aumentada de presença de anomalias, quando comparadas com 12 anos ou mais de estudo (Tabela 1). Em relação às consultas de pré-natal, observou-se que a realização de sete ou mais consultas entre os casos foi de 67\% (3.502 gestantes), enquanto, para os controles, foi de 73,6\% (15.399 gestantes). Houve associação entre o número de consultas de pré-natal e a ocorrência de anomalia congênita (valor de p $<0,001$ ) (Tabela 1).

No grupo de casos, 19,5\% tiveram abortos/perdas fetais e, no grupo controle, 16,2\%. Ter abortos/ perdas fetais previamente foi associado à ocorrência de anomalias congênitas (valor de $\mathrm{p}<0,001$ ) (Tabela 1). Em ambos os grupos, a maioria das mães não tinha filhos prévios (46,8\% nos casos e $48 \%$ nos controles) e a variável "filhos vivos" foi associada à ocorrência de anomalia congênita (valor de $\mathrm{p}<0,005)$. O fato de as mulheres já terem filhos prévios a esta gestação também se mostrou significativamente associado à ocorrência de anomalias congênitas (valor de p =0,005). 
Após o ajuste pelo modelo multivariado, permaneceram estatisticamente associados com a ocorrência de anomalias congênitas as seguintes variáveis: raça/cor, faixa etária, anos de estudo, número de consultas de pré-natal e abortos/perdas fetais prévios. Já as variáveis "filhos vivos" e "filhos prévios”, que tinham se mostrado associadas na análise univariada, saíram do modelo multivariado (valor de $\mathrm{p}>0,05$ ). Ter a cor preta aumentou em $20 \%$ a chance de anomalias congênitas, quando comparada com a cor branca $(\mathrm{OR}=1,20$; valor de $\mathrm{p}=0,013)$, e possuir mais de 40 anos aumentou em $97 \%$ a chance de anomalias congênitas, comparando-se à faixa etária de 18 a 29 anos $(\mathrm{OR}=1,97$; valor de $\mathrm{p}$ $<0,001)$. A variável anos de estudo mostrou que ter menos de 12 anos de estudo aumentou a chance de anomalias congênitas, comparando-se a 12 anos ou mais de estudo. Destaca-se que ter menos de quatro anos de estudo aumentou em $50 \%$ a chance de anomalias congênitas $(\mathrm{OR}=1,50$; valor de $\mathrm{p}=$ 0,001); não ter realizado nenhuma consulta de pré-natal aumentou a chance de anomalias congênitas quando comparada a ter realizado sete ou mais consultas, sendo que nenhuma consulta aumentou em 97\% a chance de anomalias congênitas ( $O R=1,97$; valor de $\mathrm{p}=0,001)$. A ocorrência de abortos/ perdas fetais aumentou em $17 \%$ a chance de anomalias congênitas quando comparada a nunca ter tido abortos/perdas fetais prévios $(\mathrm{OR}=1,17$; valor de $\mathrm{p}=0,001)$ (Tabela 2).

Para verificar o efeito da variável cor/raça sobre as outras variáveis "independentes" escolaridade e número de consultas pré-natais, foram utilizados os dados dos recém-nascidos sem malformação ( $\mathrm{n}=21.000)$, pois, independentemente do papel dos determinantes sociais, há também a contribuição do fator genético, que pode estar ligado à cor/raça para muitos defeitos congênitos. Nesta análise, verificou-se que existe uma associação estatisticamente significativa da variável raça/cor, tanto com

\section{Tabela 2}

Análise multivariada dos fatores independentemente associados com a ocorrência de anomalias congênitas. Porto Alegre, Rio Grande do Sul, Brasil, 2012-2015.

\begin{tabular}{|c|c|c|}
\hline Variável & OR (IC95\%) & Valor de $p$ \\
\hline Raça/Cor & & 0,013 \\
\hline Branca & 1,00 & \\
\hline Preta & $1,20(1,07-1,35)$ & \\
\hline Amarela & $1,71(0,74-3,93)$ & \\
\hline Parda & $1,03(0,92-1,15)$ & \\
\hline Indígena & $0,75(0,44-1,26)$ & \\
\hline Faixa etária (anos) & & $<0,001$ \\
\hline$<18$ & $1,03(0,90-1,18)$ & \\
\hline $18-29$ & 1,00 & \\
\hline $30-40$ & $1,17(1,08-1,26)$ & \\
\hline$>40$ & $1,97(1,63-2,39)$ & \\
\hline Anos de estudo & & $<0,001$ \\
\hline$<4$ & $1,50(1,21-1,86)$ & \\
\hline $4-7$ & $1,46(1,30-1,63)$ & \\
\hline $8-11$ & $1,36(1,24-1,49)$ & \\
\hline 12 ou mais & 1,00 & \\
\hline Consultas de pré-natal & & $<0,001$ \\
\hline Nenhuma & $1,97(1,61-2,42)$ & \\
\hline $1-3$ & $1,68(1,48-1,91)$ & \\
\hline $4-6$ & $1,23(1,14-1,34)$ & \\
\hline 7 e mais & 1,00 & \\
\hline Abortos/Perdas fetais & & $<0,001$ \\
\hline Sim & $1,17(1,07-1,27)$ & \\
\hline Não & 1,00 & \\
\hline
\end{tabular}

IC95\%: intervalo de 95\% de confiança; OR: odds ratio. 
a escolaridade como com a variável número de consultas no pré-natal (valor de $\mathrm{p}<0,001$ ). Pode-se observar, pela Tabela 3, que a relação da variável raça/cor com a escolaridade indica que tanto a cor preta como a cor parda foram associadas com baixa escolaridade. Enquanto 37,6\% das mulheres pretas e 39,9\% das pardas tiveram até sete anos de escolaridade, as mulheres brancas que tiveram essa mesma escolaridade são 22,4\% (Tabela 3). Já na avaliação da relação da variável raça/cor com o número de consultas de pré-natal (Tabela 4), foi possível observar que tanto a cor preta como a cor parda tiveram uma proporção maior de mulheres com o número insuficiente de consultas de pré-natal. Entre as mulheres pretas, 59\% tiveram o número adequado de consultas, entre as pardas, essa proporção foi de $61,8 \%$, e o número entre as brancas foi de 74,8\% (Tabela 4).

\section{Discussão}

Neste estudo, os casos de anomalias congênitas representaram 0,93\% do total de nascimentos. No Município de São Paulo, pesquisadores também utilizaram as informações contidas nas DNV e identificaram prevalência de 1,2\% de anomalias congênitas entre 2007 a 2011 10. Todavia a incidência das malformações pode ser subestimada, visto que as mais graves tendem a desencadear perdas fetais, e alguns tipos de anomalias são de difícil diagnóstico, levando à subnotificação ${ }^{11}$. Estima-se que as anomalias congênitas afetam cerca de $3 \%$ a $5 \%$ de todos os nascimentos $12,13,14$.

\section{Tabela 3}

Associação entre raça/cor e escolaridade de mães de nascidos vivos do grupo de casos de anomalias congênitas. Porto Alegre, Rio Grande do Sul, Brasil, 2012-2015 ( $\mathrm{n}=25.629)$.

\begin{tabular}{lccccc}
\hline Raça/Cor & \multicolumn{5}{c}{ Escolaridade em anos de estudo } \\
& $\mathbf{4}$ & $\mathbf{4}$ & $\mathbf{8 - 1 1}$ & $\mathbf{1 2}$ ou mais & Total \\
& $\mathbf{n ( \% )}$ & $\mathbf{n}(\mathbf{\%})$ & $\mathbf{n}(\mathbf{\%})$ & $\mathbf{n}(\mathbf{\%})$ & $\mathbf{n}(\%)$ \\
\hline Branca & $339(2,0)$ & $3.353(19,8)$ & $9.133(53,8)$ & $4.145(24,4)$ & $16.970(100,0)$ \\
Preta & $54(3,6)$ & $507(34,0)$ & $831(55,8)$ & $99(6,6)$ & $1.491(100,0)$ \\
Amarela & $0(0,0)$ & $1(5,0)$ & $14(70,0)$ & $5(25,0)$ & $20(100,0)$ \\
Parda & $85(4,4)$ & $678(35,1)$ & $1.052(54,5)$ & $115(6,0)$ & $1.930(100,0)$ \\
Indígena & $16(16,8)$ & $44(46,3)$ & $30(31,6)$ & $5(5,3)$ & $95(100,0)$ \\
Total & $494(2,4)$ & $4.583(22,3)$ & $11.060(53,9)$ & $4.369(21,3)$ & $20.506(100,0)$ \\
\hline
\end{tabular}

\section{Tabela 4}

Associação entre raça/cor e número de consultas de pré-natal de mães de nascidos vivos do grupo de casos de anomalias congênitas. Porto Alegre, Rio Grande do Sul, Brasil, 2012-2015 ( $n=25.651$ ).

\begin{tabular}{|c|c|c|c|c|c|}
\hline \multirow[t]{3}{*}{ Raça/Cor } & \multicolumn{5}{|c|}{ Número de consultas de pré-natal } \\
\hline & Nenhuma & $1-3$ & 4-6 & 7 ou mais & Total \\
\hline & n (\%) & n (\%) & n (\%) & n (\%) & n (\%) \\
\hline Branca & $276(1,6)$ & $748(4,4)$ & $3.048(18,0)$ & $12.908(76,0)$ & $16.980(100,0)$ \\
\hline Preta & $38(2,6)$ & $153(10,3)$ & $405(27,3)$ & $890(59,8)$ & $1.486(100,0)$ \\
\hline Amarela & $0(0,0)$ & $1(5,0)$ & $4(20,0)$ & $15(75,0)$ & $20(100,0)$ \\
\hline Parda & $56(2,9)$ & $165(8,6)$ & $493(25,6)$ & $1.214(62,9)$ & $1.928(100,0)$ \\
\hline Indígena & $1(1,1)$ & $8(8,4)$ & $33(34,7)$ & $53(55,8)$ & $95(100,0)$ \\
\hline Total & $371(1,8)$ & $1.075(5,2)$ & $3.983(19,4)$ & $15.080(73,5)$ & $20.509(100,0)$ \\
\hline
\end{tabular}


Neste estudo, as anomalias congênitas mais prevalentes foram as malformações e deformidades congênitas do sistema osteomuscular, que representaram $45,4 \%$ do total. Outras pesquisas corroboram com os achados neste estudo 10,15,16,17,18. A predominância de malformações osteomusculares na maioria dos estudos pode estar correlacionada à facilidade de diagnóstico, antes ou logo após o nascimento, pois são malformações macrossômicas, visíveis e facilmente detectáveis ao exame físico sumário 15. Outras tipologias, como anomalias cromossômicas não aparentes, demandam uso de maior tecnologia diagnóstica e, também, maior demanda de tempo, o que acaba resultando em diagnóstico tardio e consequente subnotificação dos casos 15,19.

No modelo dos DSS proposto por Dahlgren \& Whitehead 3, características individuais, como idade, sexo e fatores genéticos, influenciam nas condições de saúde das pessoas. Neste estudo, 80,5\% das mães de recém-nascidos com anomalia congênita no grupo de casos se autodeclararam brancas, enquanto $9,9 \%$ pardas, seguida por 9,1\% pretas. Já no grupo controle, $82,7 \%$ se autodeclararam brancas, $9,4 \%$ pardas e $7,3 \%$ pretas. A variável raça/cor foi associada à ocorrência de anomalias congênitas (valor de $\mathrm{p}<0,001)$. Na análise multivariada, ter raça/cor preta aumentou em $20 \%$ a chance de ocorrência de anomalias congênitas $(\mathrm{OR}=1,20$; valor de $\mathrm{p}=0,013)$ quando comparada à raça/cor branca. Há estudos que encontraram associações entre a ocorrência de anomalias congênitas com a raça/cor preta e parda das mães 17,20 . Outro trabalho encontrou maior prevalência das raças indígena $(11,4 / 100$ mil) e preta (10,9/100 mil) entre as mães de nascidos vivos com anomalias congênitas no Rio de Janeiro 15.

Em uma pesquisa no banco de dados da Healthcare Cost and Utilization Project - Nationwide Inpatient Sample (HCUP-NIS - https://www.hcup-us.ahrq.gov/nisoverview.jsp), nos Estados Unidos, os autores constataram que, em relação aos caucasianos, os afro-americanos apresentam menor risco de gerar descendentes com anomalias congênitas cardíacas, gênito-urinárias e craniofaciais, entretanto apresentam maior risco para o desenvolvimento de anomalias musculoesqueléticas 21. Neste estudo, as diferenças raciais/étnicas observadas, com relação ao risco de anomalias congênitas, podem estar relacionadas a diversos fatores, como a suscetibilidade genética e as diferenças culturais ou sociais, que podem modificar as exposições, como nível socioeconômico, nutrição, estresse e acesso aos cuidados médicos, ou muitas combinações potenciais entre as suscetibilidades e as exposições 21,22.

No Brasil, mais da metade das pessoas (53,9\%) se declararam de raça/cor preta ou parda no censo de 2010 realizado pelo Instituto Brasileiro de Geografia e Estatística (IBGE). Contudo, a proporção de pessoas que se declararam pretas ou pardas no Estado do Rio Grande do Sul foi de apenas 22,5\% 23. Para os campos da psicologia social e da saúde coletiva, fica o desafio de qualificar a notificação do quesito raça/cor, inaugurando um novo processo de discussão da temática racial e do enfrentamento ao racismo estrutural e institucional, no âmbito do Sistema Único de Saúde (SUS) 24. Para enfrentar tais limitações, é preciso entender um pouco mais sobre as formas como o racismo opera na sociedade. É preciso destacar que o racismo e a longa trajetória de discriminações, acrescidos da precariedade e baixa qualidade dos serviços destinados a determinadas camadas da população, interpõem-se entre profissionais e usuários, influenciando visões preconceituosas e estereotipadas por parte de profissionais em relação a integrantes de grupos subordinados, e dificultando o estabelecimento de relações de confiança, tanto entre profissionais e usuários quanto entre usuários e os serviços, e todo o sistema de saúde 25 .

Neste estudo, a faixa etária predominante em ambos os grupos (casos e controles) foi de 18 a 29 anos, com 51,8\% e 55,1\%, respectivamente; e, na análise multivariada, possuir mais de 40 anos aumentou em 97\% a chance de anomalias congênitas, comparando-se à faixa etária de 18 a 29 anos $(\mathrm{OR}=1,97$; valor de $\mathrm{p}<0,001)$. A idade materna avançada também é fator de risco para anormalidades no desenvolvimento fetal intrauterino e aumenta o risco de anormalidades cromossômicas, incluindo a síndrome de Down 1.

A faixa etária de homens e mulheres que decidem ter filhos aumentou consideravelmente nos países desenvolvidos, elevando o surgimento de anomalias congênitas relacionadas com a idade. Pesquisas referem que mulheres em idades mais avançadas têm mais chances de ter filhos com síndromes ou outras anomalias cromossômicas 26,27,28. Em estudo de dados do Medical Birth Register (2000-2010), na Letônia, pesquisadores observaram que a prevalência de anomalias congênitas aumentou conforme se elevou a idade materna; defeitos cardíacos congênitos, defeitos nos membros e anomalias no sistema urinário foram as anomalias mais comuns 29 . 
Na segunda camada do modelo de Dahlgren \& Whitehead 3, estão os comportamentos e os estilos de vida individuais. Embora muitas vezes considerados como escolhas de responsabilidade individual, os comportamentos são muito afetados pelos DSS, pois estão também condicionados por determinantes sociais, como informações, propagandas, possibilidades de acesso a alimentos saudáveis e espaços de lazer, entre outros. Neste estudo, tanto no grupo de casos quanto no grupo de controles, a maioria das mães não tinha filhos prévios (46,8\% e 48\%, respetivamente). O fato de possuir três ou mais filhos foi associado à ocorrência de anomalia congênita (valor de p < 0,005). Acredita-se que quanto mais filhos a mulher gere, maior idade ela terá e, neste sentido, fatores biológicos poderão interferir na relação entre maior número de filhos e a ocorrência de anomalia congênita.

Em estudo com dados secundários do SINASC sobre anomalia congênita no Nordeste brasileiro, observou-se que a maioria das mulheres possuía filhos prévios $(65,1 \%)$ e apresentava multiplicidade de gestações anteriores com quatro ou mais gestações $(93,1 \%)$, ambas as características tendo sido associadas à ocorrência de anomalia congênita 18. Por outro lado, um estudo sobre anomalia congênita no sul da Índia associou primigestas ao maior risco de ocorrência de anomalia congênita 30 . Há ainda outra pesquisa, realizada nos municípios do Vale do Paraíba paulista, que não encontrou associação entre as malformações e o número de gestações 31. As pesquisas realizadas não encontraram consenso quanto à relação do número de gestações anteriores e a ocorrência de anomalias congênitas.

No grupo de casos deste estudo, 19,5\% das mulheres tiveram abortos/perdas fetais e, no grupo controle, 16,2\%. A ocorrência de abortos/perdas fetais foi associada à anomalias congênitas (valor de $\mathrm{p}$ $<0,001)$ e, na análise multivariada, essa condição aumentou em 17\% a chance de anomalias congênitas, comparando-se a nunca ter tido abortos/perdas fetais prévios $(\mathrm{OR}=1,17$; valor de $\mathrm{p}<0,001)$. Outros autores, entretanto, não encontraram associação entre antecedentes de abortos e as malformações fetais 32 . Assim, como o número de gestações anteriores, ter tido aborto também não mostra consenso entre as pesquisas já realizadas, embora se acredite que mulheres que geraram filhos com anomalias congênitas e apresentaram abortos anteriores podem ter gerado embriões ou fetos com anomalias congênitas incompatíveis com a vida.

Em um nível mais distal do modelo de Dahlgren \& Whitehead 3, estão destacados os fatores relacionados às condições de vida e de trabalho, disponibilidade de alimentos e, também, acesso a ambientes e serviços essenciais, como saúde e educação. Esses indicadores evidenciam que as pessoas em desvantagem social têm risco diferenciado em saúde, criado por condições habitacionais precárias, exposição a condições perigosas ou estressantes de trabalho e menor acesso aos serviços. Em relação à variável escolaridade analisada neste estudo, o fator ter menos de sete anos de estudo foi associado à ocorrência de anomalias congênitas (valor de $\mathrm{p}<0,000$ ). Na análise multivariada, ter menos de quatro anos de estudo aumentou em 50\% a chance de anomalias congênitas, comparando-se a ter 12 anos ou mais de estudo $(\mathrm{OR}=1,50$; valor de $\mathrm{p}<0,001)$. Há outras pesquisas que também relacionaram a menor escolaridade com maior ocorrência de casos de anomalias congênitas 17,20,28. Quanto maior a escolaridade das mães, maior o discernimento em relação à necessidade de acompanhamento da sua gestação e aos fatores de riscos. Isso pode aumentar as chances da gestante entender e utilizar suplementação de ácido fólico, por exemplo, e assim, prevenir defeitos relacionados ao nascimento e complicações comuns da gravidez ${ }^{17}$. Em um estudo na Colômbia, observou-se associação entre a não realização de ultrassonografias durante o pré-natal e mães com baixa escolaridade, todavia a associação mais robusta foi entre as mães sem Ensino Fundamental completo $(\mathrm{OR}=8,70)$ 33. É importante ressaltar que, muitas vezes, a baixa escolaridade está diretamente relacionada ao perfil socioeconômico, devendo-se levar em conta o impacto do nascimento de uma criança malformada para uma família com menores recursos econômicos, pois o recém nascido não pode ser visto separadamente da mãe, ou melhor, de sua família e contexto social 15 .

A realização de Ensino Superior na faixa etária de 18 a 24 anos ainda é bastante desigual, dependendo das características de cor ou raça no Brasil. O total de pessoas de cor preta ou parda dessa faixa etária que cursavam o Ensino Superior foi de 12,8\% em 2015. Esse percentual representa um crescimento significativo em relação a 2005 (7,3 pontos percentuais), mas ainda ficou abaixo do percentual alcançado pelos jovens estudantes brancos 10 anos antes $(17,8 \%) 23$.

Quanto às consultas de pré-natal, a realização de sete ou mais consultas entre os casos foi de 67\% (3.502 gestantes), enquanto para os controles foi de 73,6\% (15.399 gestantes). Houve associação entre o número de consultas de pré-natal e a ocorrência de anomalia congênita (valor de $\mathrm{p}<0,001$ ). 
O fato de a gestante não ter realizado nenhuma consulta de pré-natal aumentou em 97\% a chance de anomalias congênitas, quando comparado a ter realizado sete ou mais consultas $(\mathrm{OR}=1,97$; valor de $\mathrm{p}<0,001)$. Outros trabalhos também observaram maior prevalência de malformações entre as mulheres que realizaram menos consultas pré-natais 15,17 . Uma pesquisa descreveu que a razão de prevalência de microcefalia ao nascer foi 2,6 vezes maior entre mães que não realizaram o pré-natal em relação àquelas com seis ou mais consultas, e os coeficientes de prevalência de microcefalia foram menores entre nascidos de mães com maior número de consultas de pré-natal 20. Outro estudo, no entanto, não encontrou significância estatística entre as malformações congênitas e o número de consultas de pré-natal 18. O modelo de atenção pré-natal proposto pela Organização Mundial da Saúde (OMS) recomenda a realização de oito consultas 34 . O pré-natal é, portanto, um conjunto de medidas essenciais à redução da morbimortalidade e da promoção do bem-estar materno-infantil 35,36.

Algumas das causas de anomalias congênitas como fatores genéticos, infecciosos, nutricionais ou ambientais são conhecidas, porém, muitas vezes, é difícil identificar as causas exatas. A vacinação, a ingestão adequada de ácido fólico ou iodo, por meio da fortificação de alimentos básicos ou suplementação, e cuidados pré-natais adequados, são exemplos de métodos de prevenção 1. Ao avaliar 29 casos de síndrome congênita do Zika em uma região de Pernambuco, pesquisadores afirmaram que $20 \%$ das mães relataram não ter tomado a vacina contra rubéola e sarampo ${ }^{37}$.

Em um estudo com mulheres que tiveram filhos no ano de 2013 no Estado do Rio Grande do Sul, encontrou-se a presença de iniquidade em saúde de forma contrária ao esperado ${ }^{38}$. Os autores observaram maior cobertura de suplementação de sulfato ferroso entre as mães mais jovens e de baixa escolaridade (justamente as mais vulneráveis a apresentar pior desempenho em termos de desfecho gestacional), com renda familiar inferior a dois salários mínimos e que utilizaram o sistema público de pré-natal. Observa-se, neste estudo, um exemplo de equidade, visto que os grupos menos favorecidos foram os mais privilegiados. No entanto os autores chamam atenção para o fato de que as políticas públicas não foram feitas para atender apenas aos mais pobres, e sim a toda a população, afinal, a recomendação de suplementação de sulfato ferroso pelo Ministério da Saúde é direcionada a todas as gestantes, independentemente dos fatores socioeconômicos 38,39 .

Este estudo também observou uma associação da raça/cor preta com a baixa escolaridade e com menor número de consultas de pré-natal (valor de $\mathrm{p}<0,001$ ). O mesmo tipo de associação também aconteceu na raça/cor parda. Para a variável escolaridade, ainda foi encontrada associação significativa entre a raça/cor indígena. Destacam-se, pela OMS, desvantagens para as pessoas de cor preta ou parda, com a expressiva maioria sem plano de saúde (78,8\%), menor acesso à saúde e maior exposição a riscos. Pessoas com rendimentos inferiores, sem acesso à educação e em condições de moradia precárias por falta de acesso a serviços básicos também se mostram mais expostas. Ainda, as desigualdades poderiam ser minimizadas a partir de políticas direcionadas, sem esquecer que as vulnerabilidades tendem a se sobrepor, com acúmulo de desvantagens para alguns grupos - em geral, as pessoas de raça/cor preta e parda 23 .

Ao analisar bases de dados secundários no Rio Grande do Sul de 2005 a 2014, pesquisadores encontraram maiores taxas de anomalias congênitas entre as mães com idade maior ou igual a 40 anos, de cor preta ou parda e que realizaram menos de três consultas de pré-natal 39 . É necessário que se estabeleçam medidas facilitadoras da aproximação e acesso, a fim de superar as barreiras interpostas ao exercício do direito à saúde pelas mulheres negras 25 .

Em um estudo que analisou as mães de recém-nascidos com e sem anomalias cardíacas congênitas, em uma base de dados secundários da Califórnia (Estados Unidos), comparou-se a etnia hispânica à etnia branca não hispânica e identificou-se que a etnia hispânica foi associada à anomalias congênitas, enquanto a maior escolaridade e o fato de possuir convênio de saúde foram identificados como fatores de proteção 40 . Os autores concluíram que os fatores socioeconômicos explicaram uma parcela significativa da associação entre a etnia hispânica e anomalias congênitas em neonatos, indicando que há fatores específicos dentro de grupos étnicos/raciais que podem ser alvo de intervenções estratégicas para minimizar as disparidades em saúde, como, por exemplo, identificar as barreiras do acesso aos serviços de saúde 40 .

Este estudo colaborou na geração de conhecimento e fomentou a discussão da influência dos DSS na ocorrência de anomalias congênitas no Estado do Rio Grande do Sul, demonstrando a necessidade de dar visibilidade a elementos importantes, como acesso a um pré-natal mais inclusivo, capaz de 
acompanhar mulheres que vivam em situações de vida desfavorável, bem como o enfrentamento ao racismo institucional presente nos serviços de saúde, que excluem as mulheres de raça/cor preta ou parda de um acompanhamento adequado. Por fim, espera-se que este estudo possa auxiliar na garantia de políticas de ações afirmativas que propiciem mais oportunidades e melhorem as condições de vida, renda e saúde das mulheres.

\section{Conclusão}

Este estudo buscou analisar os fatores associados aos casos de anomalias congênitas na perspectiva dos DSS no Rio Grande do Sul. Os resultados encontrados trazem à discussão as desigualdades raciais e sociais, relacionando-as direta ou indiretamente às iniquidades em saúde. Enfatiza-se que, principalmente em estudos observacionais, a correlação não é causalidade. Fatores no ambiente social, dentre eles o racismo institucional, determinam o acesso a serviços de saúde e as escolhas no estilo de vida do indivíduo, pois os comportamentos de risco são influenciados pelas normas culturais e pelas oportunidades ao longo da vida. Os profissionais de saúde devem ser capazes de observar a mulher em idade fértil, acompanhar as condições de vida e saúde da gestante, considerando com atenção os fatores de risco e as causas preveníveis das anomalias congênitas.

As limitações deste estudo concernem ao próprio documento utilizado para análise (DNV), que impediu análises de outras possíveis variáveis além das que constam no documento. É preciso que se perceba a influência do ambiente na saúde da população, considerando não apenas fatores biológicos, mas também questões raciais, sociais, condições de vida e trabalho, processos de adoecimento e vulnerabilidades.

\section{Colaboradores}

G. C. Trevilato, D. L. Riquinho, M. O. Mesquita, I. Rosset, L. G. S. Augusto e L. N. Nunes contribuíram na concepção do estudo, coleta de dados, análise e interpretação dos dados, redação e revisão crítica do conteúdo, na revisão e aprovação da versão final e são responsáveis por todos os aspectos do trabalho.

\section{Informações adicionais}

ORCID: Graziella Chaves Trevilato (0000-00022700-5587); Deise Lisboa Riquinho (0000-00026604-8985); Marilise Oliveira Mesquita (00000002-4415-2386); Idiane Rosset (0000-0003-3651652X); Lia Giraldo da Silva Augusto (0000-00029322-6863); Luciana Neves Nunes (0000-00030151-1876).

\section{Agradecimentos}

Ao Sistema de Informações sobre Nascidos Vivos do Estado do Rio Grande do Sul (SINASC/RS) pela colaboração no fornecimento dos dados, ao Programa de Pós-graduação em Enfermagem da Escola de Enfermagem da Universidade Federal do Rio Grande do Sul pelo apoio e suporte durante a realização da pesquisa, e ao Conselho Nacional de Desenvolvimento Científico e Tecnológico (CNPq, processo no 427983/2018-5) pelo apoio financeiro. 


\section{Referências}

1. World Health Organization. Congenital anomalies, 2016. https://www.who.int/en/newsroom/fact-sheets/detail/congenital-anomalies (acessado em 5/Out/2016).

2. Pan American Health Organization; World Bank. Present and future of the surveillance of congenital defects in the Americas. https://iris. paho.org/handle/10665.2/51964 (acessado em 21/Jun/2021).

3. Dahlgren G, Whitehead M. Policies and strategies to promote social equity in health. Stockholm: Institute for Future Studies; 1991.

4. Maeyama MA, Jasper CH, Nilson LG, Dolny LL, Cutolo LRA. Promoção da saúde como tecnologia para transformação social. Revista Brasileira de Tecnologias Sociais 2015; 2:129-43.

5. Rocha PR, David HMSL. Determinação ou determinantes? Uma discussão com base na teoria da produção social da saúde. Rev Esc Enferm USP 2015; 49:129-35.

6. Almeida AR, Athayde FTS. Promoção da saúde, qualidade de vida e iniquidade em saúde: reflexões para a saúde pública. Tempus (Brasília) 2015; 9:165-72.

7. Paixão M, Rossetto I, Montovanele F, Carvano LM. Relatório anual das desigualdades raciais no Brasil; 2009-2010. Rio de Janeiro: Garamond; 2010

8. Newman TB, Browner WS, Cummings SR, Hulley SB. Delineando estudos transversais e de caso-controle. In: Hulley SB, Cummings SR, Browner WS, Grady D, Herasyt N, Newman TB, organizadores. Delineando pesquisa clínica: uma abordagem epidemiológica. Porto Alegre: Artmed; 2008. p. 130-9.

9. Departamento de Informática do SUS. Consolidação do Sistema de Informações sobre Nascidos Vivos, 2011. http://tabnet.datasus. gov.br/cgi/sinasc/Consolida_Sinasc_2011.pdf (acessado em 03/Out/2016).

10. Mendes CQS, Avena MJ, Mandetta MA, Balieiro MMFG. Prevalência de nascidos vivos com anomalias congênitas no município de São Paulo. Rev Soc Bras Enferm Pediatras 2015; 15:7-12.

11. Departamento de Análise de Situação de Saúde, Secretaria de Vigilância em Saúde, Ministério da Saúde. Manual de instruções para o preenchimento da declaração de nascido vivo. http://portalarquivos.saude.gov.br/images/ pdf/2017/junho/08/inst_dn.pdf (acessado em 05/Dez/2019).

12. García AM, Fletcher T, Benavides FG, Orts E. Parental agricultural work and selected congenital malformations. Am J Epidemiol 1999; 149:64-74.

13. Horovitz DDG, Llerena Jr. JC, Mattos RA. Atenção aos defeitos congênitos no Brasil: panorama atual. Cad Saúde Pública 2005; 21:1055-64.

14. Benítez-leite S, Macchi ML, Acosta M. Malformaciones congénitas associadas a agrotóxicos. Arch Pediatr Urug 2009; 80:237-47.
15. Reis AT, Santos RS, Mendes TAR. Prevalência de malformações congênitas no Município do Rio de Janeiro, Brasil, entre 2000 e 2006. Rev Enferm UERJ 2011; 19:364-8.

16. Cosme HW, Lima LS, Barbosa LG. Prevalência de anomalias congênitas e fatores associados em recém-nascidos do Município de São Paulo no período de 2010 a 2014. Rev Paul Pediatr 2017; 35:33-8.

17. Rodrigues LS, Lima RHS, Costa LC, Batista RFL. Características das crianças nascidas com malformações congênitas no município de São Luís, Maranhão, 2002-2011. Epidemiol Serv Saúde 2014; 23:295-304.

18. Melo RA, Fernandes FECV, Araújo AKC, Santos NM, Campos MEAL, Sobral PHAF, et al. Congenital malformations in neonates: analysis of morbidity and associated factors. Int Arch Med 2017; 10:1-8.

19. Braz P, Machado A, Dias CM. Registo racional de anomalias congênitas: relatório de 2011-2013. https://core.ac.uk/download/ pdf/70642130.pdf (acessado em 13/Abr/2019).

20. Marinho F, Araújo VEMD, Porto DL, Ferreira HL, Coelho MRS, Lecca RCR, et al. Microcephaly in Brazil: prevalence and characterization of cases from the Information System on Live Births (Sinasc), 2000-2015. Epidemiol Serv Saúde 2016; 25:701-12.

21. Egbe A, Lee S, Ho D, Uppu S, Srivastava S. Racial/ethnic differences in the birth prevalence of congenital anomalies in the United States. J Perinat Med 2015; 43:111-7.

22. Egbe A, Lee S, Ho D, Uppu S. Effect of race on the prevalence of congenital malformations among newborns in the united states. Ethn Dis 2015; 25:226-31.

23. Instituto Brasileiro de Geografia e Estatística. Síntese de indicadores sociais: uma análise das condições de vida da população brasileira. Rio de Janeiro: Instituto Brasileiro de Geografia e Estatística; 2016. (Estudos e Pesquisas. Informação Demográfica e Socioeconômica, 39).

24. Alves MC, Jesus JP, Diaz LAF. Autodeclaração da raça/cor no SUS: reflexões conceituais a partir da campanha realizada pelo estado do Rio Grande do Sul. Revista Identidade 2017; 22:5-15.

25. Werneck J. Racismo institucional e saúde da população negra. Saúde Soc 2016; 25:535-49.

26. Miller A, Riehle-Colarusso T, Siffel C, Frías JL, Correa A. Maternal age and prevalence of isolated congenital heart defects in an urban area of the United States. Am J Med Genet A 2011; 155A:2137-45.

27. Herkrath APCQ, Herkrath FJ, Rebelo MAB, Vettore MV. Parental age as a risk factor for non-syndromic oral clefts: a meta-analysis. J Dent 2012; 40:3-14.

28. Rychtarikova J, Gourbin, C, Wunsch G, Sipek A. Impact of parental ages and other characteristics at childbearing on congenital anomalies: results for the Czech Republic, 2000-2007. Demogr Res 2013; 28:137-76. 
29. Zile I, Villerusa A. Maternal age-associated congenital anomalies among newborns: a retrospective study in Latvia. Medicina (Kaunas) 2013; 49:29-35.

30. Sunitha 1, Prasoona KR, Kumari TM, Srinadh B, Deepika NML, Aruna R, et al. Risk factors for congenital anomalies in high risk pregnant women: a large study from South India. Egyptian Journal of Medical Human Genetics 2017; 18:79-85.

31. Pinto CO, Nascimento LFC. Estudo de prevalência de defeitos congênitos no Vale do Paraíba Paulista. Rev Paul Pediatr 2007; 25:233-9.

32. Rizk F, Salameh P, Hamadé A. Congenital anomalies: prevalence and risk factors. Univers J Public Health 2014; 2:58-63.

33. Saldarriga Gil W, Ruiz Murcia FA, Fandinõlosada A, Cruz Perea ME, Isaza de Lourido C. Evaluación del diagnóstico prenatal de defectos congénitos por ecografía de tamizaje, en Cali, Colombia. Colomb Méd 2014; 45:32-8.

34. Organização Mundial da Saúde. OMS publica novas orientações sobre pré-natal para reduzir mortes de mães e bebês. https://nacoesunidas. org/oms-publica-novas-orientacoes-sobre -pre-natal-para-reduzir-mortes-de-maes-e -bebes/ (acessado em 14/Abr/2019).

35. Gomes RMT, César JA. Perfil epidemiológico de gestantes e qualidade do pré-natal em unidade básica de saúde em Porto Alegre, Rio Grande do Sul, Brasil. Rev Bras Med Fam Comunidade 2013; 8:80-9.
36. Migoto TM, Oliveira RP, Andrade L, Freire MHS. Correlação espacial da mortalidade perinatal com condições sociais, econômicas e demográficas: estudo ecológico. Rev Saúde Pública Paraná 2020; 3:75-85.

37. Lopes BC, Bezerra DWG, Briano MLF, Ferreira Júnior DL, Soares ENL. A influência dos determinantes sociais em saúde nos casos de síndrome congênita associada à infecção pelo vírus Zika ocorridos na IV Região de Saúde de Pernambuco. Caruaru: Associação Caruaruense de Ensino Superior e Técnico; 2018.

38. Linhares AO, Linhares RS, Cesar JA. Iniquidade na suplementação de sulfato ferroso entre gestantes no sul do Brasil. Rev Bras Epidemiol 2017; 20:650-60.

39. Luz GS, Karam SM, Dumith SC. Anomalias congênitas no estado do Rio Grande do Sul: análise de série temporal. Rev Bras Epidemiol 2019; 22:E190040.

40. Peyvandi S, Baer RJ, Moon-grady AJ, Oltman $\mathrm{SO}$, Chambers CD, Norton ME, et al. Socioeconomic mediators of racial and ethnic disparities in congenital heart disease outcomes: a population-based study in California. J Am Heart Assoc 2018; 7:e010342. 


\section{Abstract}

The objective of this study was to analyze factors associated with cases of congenital anomalies from the perspective of social determinants of health in the State of Rio Grande do Sul, Brazil. This is a case-control study with all the pairs of mothers and liveborn infants from 2012 to 2015 in the state, based on the total number of liveborn infants with congenital anomalies $(5,250)$ and a random sample of 21,000 without congenital anomalies, according to data on the live birth certificates. The statistical analyses included chi-square tests and logistic regression models with SPSS. The Dahlgren ef Whitehead model was used as the basis for grouping and discussing the variables. In the multivariate model, all the variables that were significantly associated with the outcome were in the sense of increasing the odds of births with congenital anomalies: black women had 20\% higher odds than white women $(O R=1.20$; $p$-value $=$ $0.013) ;$ age over 40 years increased the odds by 97\% when compared to women 18 to 29 years of age; women with less than four years of schooling showed $50 \%$ higher odds when compared to women with 12 or more years of schooling $(O R=1.50$; p-value $=0.001)$; women with no prenatal visits had $97 \%$ higher odds than women with seven or more prenatal visits $(O R=1.97$; $p$-value $=0.001)$; and prior history of miscarriages/stillbirths increased the odds by $17 \%(O R=1.17 ;$ p-value $=$ 0.001 ). The results raise the issue of racial and social inequalities, related to health inequities.

Congenital Abnormalities; Social Determinants of Health; Race Factors; Racism

\section{Resumen}

El objetivo de este estudio fue analizar los factores asociados con los casos de anomalías congénitas desde la perspectiva de los determinantes sociales en salud del estado de Rio Grande do Sul, Brasil. Se trata de un estudio de tipo caso-control, con todas las parejas de madres y nacidos vivos en el periodo de 2012 a 2015 en el estado, considerando el total de nacidos vivos con anomalía congénita (5.250), y realizando una muestra aleatoria con 21.000 sin anomalía congénita, conforme la información de las Declaraciones de Nacidos Vivos. Para el análisis estadístico, se realizaron tests chicuadrado y modelos de regresión logística mediante el SPSS. El modelo de Dahlgren es Whitehead se utilizó como base para el agrupamiento y discusión de las variables. En el modelo multivariado, todas las variables que se mostraron asociadas significativamente con el resultado fueron en el sentido de aumentar la oportunidad de nacimientos con anomalía congénita: las mujeres negras tuvieron un $20 \%$ más de oportunidad, comparadas con las blancas $(O R=1,20$; valor de $p=0,013)$; tener más de 40 años aumentó en un $97 \%$ la oportunidad, cuando se comparan con las de 18 a 29 años; las mujeres con menos de cuatro años de estudio presentaron un 50\% más de oportunidades, comparadas con las mujeres con 12 años o más de estudio ( $O R=1,50$; valor de $p=0,001)$; las mujeres que no realizaron ninguna consulta prenatal tuvieron 97\% más oportunidades, comparadas con las mujeres que realizaron siete o más consultas $(\mathrm{OR}=1,97$; valor de $p=0,001)$; y la ocurrencia de abortos/pérdidas fetales aumentó en un $17 \%$ la oportunidad, en relación a nunca haber sufrido abortos/pérdidas fetales previas $(O R=1,17$; valor de $p=0,001$ ). Los resultados plantean la discusión de las desigualdades raciales y sociales, relacionándolas con las inequidades en salud.

Anomalias Congénitas; Determinantes Sociales de la Salud; Factores Raciales; Racismo

Recebido em 13/Fev/2021

Versão final reapresentada em 25/Jun/2021

Aprovado em 08/Jul/2021 